\title{
SINGULAR PERTURBATION PROBLEMS WITH A COMPACT SUPPORT SEMILINEAR TERM
}

\author{
By \\ Giovanni Alberti \\ Luigi Ambrosio \\ and \\ Giuseppe Buttazzo
}

IMA Preprint Series \# 752

December 1990 
The meaning of the constraint condition on $B$ in (2) is the following: if the set $\{u=0\}$ is negligible, then the only admissible choice for $B$ is $B=\{u>0\}$, which gives

$$
F(u)=2\left(\int_{-\infty}^{+\infty} \beta^{1 / 2}(s) d s\right) P(\{u>0\}, \Omega) .
$$

On the contrary, if the set $\{u=0\}$ is not negligible, then we have to choose a subset $H$ of its in order to get, for $B=\{u>0\} \cup H$, the least perimeter $P(B, \Omega)$.

We also study (see Theorem 1.1 and Proposition 1.6) the asymptotic behaviour of the minimizers of the problems

$$
\int_{\Omega}\left[\varepsilon|\nabla u|^{2}+\varepsilon^{-3} \beta\left(\frac{u}{\varepsilon}\right)\right] d x+\int_{\Omega} g(x, u) d x
$$

for suitable functions $g: \Omega \times \mathbf{R} \rightarrow \mathbf{R}$.

\section{Statement of the Results}

Let $\Omega$ be a bounded open subset of $\mathbf{R}^{n}$ with a Lipschitz boundary. As usual we denote by $H^{1}(\Omega)$ the Sobolev space of all functions $u \in L^{2}(\Omega)$ with distributional derivatives in $L^{2}(\Omega)$. Let $\beta: \mathbf{R} \rightarrow \mathbf{R}$ be a nonnegative lower semicontinuous function with compact support and let $g: \Omega \times \mathbf{R} \rightarrow \mathbf{R}$ be a Borel function such that

(i) for a.e. $x \in \Omega$ the function $g(x, \cdot)$ is continuous on $\mathbf{R}$;

(ii) there exist $p_{1}, p_{2} \in L^{1}(\Omega)$ and $C_{1}, C_{2}>0$ such that for a.e. $x \in \Omega$ and every $s \in \mathbf{R}$

$$
p_{1}(x)+C_{1}|s|^{2} \leq g(x, s) \leq p_{2}(x)+C_{2}|s|^{2}
$$

Then, for every $\varepsilon>0$, the well-known direct method of the Calculus of Variations (see for instance [6]) ensures the existence of a solution $u_{\varepsilon}$ of the problem

$$
\min \left\{\int_{\Omega}\left[\varepsilon|\nabla u|^{2}+\varepsilon^{-3} \beta\left(\frac{u}{\varepsilon}\right)\right] d x+\int_{\Omega} g(x, u) d x: u \in H^{1}(\Omega)\right\} .
$$

The present paper is devoted to the characterization of the asymptotic behaviour of the solutions $u_{\varepsilon}$ and of the minimum values $\min \left(\mathcal{P}_{\varepsilon}\right)$ as $\varepsilon \rightarrow 0$. In order to describe the limit problem, we introduce the functions

$$
g_{-}(x)=\min \{g(x, t): t \leq 0\}, \quad g_{+}(x)=\min \{g(x, t): t \geq 0\},
$$

we set

$$
c=2 \int_{-\infty}^{+\infty} \beta^{1 / 2}(s) d s
$$


and we recall some basic notions about functions of bounded variation and sets of finite perimeter.

We say that $u \in L^{1}(\Omega)$ is a function with bounded variation if

$$
|D u|(\Omega)=\sup \left\{\int_{\Omega} u \operatorname{div} g d x: g \in C_{0}^{1}\left(\Omega ; \mathbf{R}^{n}\right),|g| \leq 1\right\}<+\infty .
$$

The number defined in (1.1) is called total variation of $u$ in $\Omega$, and is equal to the integral of $|\nabla u|$ if $u$ is a Lipschitz function. We denote by $B V(\Omega)$ the vector space of all functions $u$ such that $|D u|(\Omega)<+\infty$. By using Riesz's representation theorem, it can be seen that $u \in B V(\Omega)$ if and only if there is a vector measure $\left(\mu_{1}, \ldots, \mu_{n}\right)$ with finite total variation which allows integral representation of the distributional derivative of $u$, i.e.

$$
\int_{\Omega} u \frac{\partial g}{\partial x_{i}} d x=-\int_{\Omega} g d \mu_{i} \quad \forall g \in C_{0}^{1}(\Omega), i=1, \ldots, n
$$

If $u=\chi_{B}$ is the characteristic function of a Borel set $B$, then the total variation of $u$ is called perimeter of $B$ in $\Omega$, and denoted by $P(B, \Omega)$. Sets of finite perimeter have proved to be very useful in the theory of minimal surfaces. A comprehensive treatment of this topic can be found for istance in [8] and [10].

Let $(\mathcal{P})$ be the following problem

$$
\min \left\{c P(B, \Omega)+\int_{B} g_{+} d x+\int_{\Omega \backslash B} g_{-} d x: B \text { Borel set }\right\} .
$$

Well known compactness and lower semicontinuity properties of sets of finite perimeter (see for instance Giusti [10]) ensure that the infimum of $(\mathcal{P})$ is attained. The following theorem is the main result of this paper.

Theorem 1.1. We have

$$
\lim _{\varepsilon \rightarrow 0^{+}}\left[\min \left(\mathcal{P}_{\varepsilon}\right)\right]=\min (\mathcal{P})
$$

Moreover, given any sequence $\left(\varepsilon_{h}\right)$ converging to 0 and any sequence $\left(u_{h}\right)$ of solutions of $\left(\mathcal{P}_{\varepsilon_{h}}\right)$, there exist a subsequence $\left(u_{h_{k}}\right)$ and a minimizer $B$ of $(\mathcal{P})$ such that, for almost every $x \in \Omega$, either $u_{h_{k}}(x)$ converges to 0 , or $\operatorname{sign} u_{h_{k}}(x)$ converges to $\chi_{B}(x)-\chi_{\Omega \backslash B}(x)$. Finally, if

$$
g_{-}(x)<g(x, 0) \text { and } g_{+}(x)<g(x, 0) \quad \text { a.e. in } \Omega \text {, }
$$

then

$$
\operatorname{sign}\left(u_{h_{k}}\right) \rightarrow \chi_{B}-\chi_{\Omega \backslash B} \quad \text { a.e. in } \Omega \text {. }
$$


Remark 1.2. By using (ii) and the projection theorem (see for instance Castaing \& Valadier [7], Theorem III.22), it can be shown that $g_{-}$and $g_{+}$belong to $L^{1}(\Omega)$. Moreover, Aumann's measurable selection theorem (see [7], Theorem III.23) allows us to find two functions $a, b \in$ $L^{2}(\Omega)$ such that $a \leq 0, b \geq 0$ and

$$
g_{-}(x)=g(x, a(x)) \quad \text { and } \quad g_{+}(x)=g(x, b(x)) \quad \text { for a.e. } x \in \Omega \text {. }
$$

Notice that, if for every $h \in \mathbf{N}$ we set $a_{h}=(a \wedge h) \vee-h$ and $\left(b_{h} \wedge h\right) \vee-h$, we have

$$
g_{-}(x)=\lim _{h \rightarrow+\infty} g\left(x, a_{h}(x)\right) \quad \text { and } \quad g_{+}(x)=\lim _{h \rightarrow+\infty} g\left(x, b_{h}(x)\right)
$$

in the strong convergence of $L^{1}(\Omega)$.

If we denote by $\mathcal{M}(\Omega)$ the space of all real valued Borel functions defined in $\Omega$ and endow $\mathcal{M}(\Omega)$ with a distance inducing convergence in measure, then Theorem 1.1 can be partially rephrased in terms of $\Gamma$-convergence (see for instance [2] for the basic definitions) as follows.

Theorem 1.3. The functionals

$$
F_{\varepsilon}(u)= \begin{cases}\int_{\Omega}\left[\varepsilon|\nabla u|^{2}+\varepsilon^{-3} \beta\left(\frac{u_{\varepsilon}}{\varepsilon}\right)\right] d x & \text { if } u \in H^{1}(\Omega) \\ +\infty & \text { if } u \in \mathcal{M}(\Omega) \backslash H^{1}(\Omega)\end{cases}
$$

$\Gamma$-converge in $\mathcal{M}(\Omega)$, as $\varepsilon \rightarrow 0$, to the functional

$$
F(u)=\min \{c P(B, \Omega):\{u>0\} \subset B \subset\{u \geq 0\}\} .
$$

It is well-known that $\Gamma$-convergence and equicoercivity ensure convergence of minimizers to minimizers and of minimum values to minimum values. However, it can be easily seen that the functionals $F_{\varepsilon}$ are not equicoercive in $\mathcal{M}(\Omega)$. For instance, if $\Omega$ is the interval ] $0,1[$ and $u_{\varepsilon}(x)=2+\sin (x / \sqrt{\varepsilon})$, for every $\varepsilon>0$ we have $F_{\varepsilon}(u) \leq C$ for a suitable constant $C$, but it is impossible to extract subsequences $\left(u_{\varepsilon_{h}}\right)$ which converge almost everywhere. In particular, Theorem 1.1 cannot be deduced from Theorem 1.3. In order to prove (1.2) we need to consider a weaker form of convergence (see Proposition 2.1).

Remark 1.4. Since the perimeter $P(B, \Omega)$ is not affected by modifications of $B$ in negligible sets, an equivalent definition of $F$ can be given by requiring the inclusions to hold only almost everywhere. We also remark that $F(u)=c P(\{u>0\}, \Omega)$ if $u \neq 0$ almost everywhere, and $F(u)=0$ if either $u \geq 0$ or $u \leq 0$ in $\Omega$.

Remark 1.5. When we consider $F_{\varepsilon}$ as functions also of the the domain of integration $\Omega$ (and then we write $F_{\varepsilon}(u, \Omega)$ instead of $\left.F_{\varepsilon}(u)\right)$, it is interesting to notice that even if all 
the functionals $F_{\varepsilon}$ are $\sigma$-additive measures as functions of $\Omega$, the $\Gamma$-limit $F$ does not share this property. In the one dimensional case, it suffices to take a function $u$ equal to 1 in ] $0,1 / 3$ [ , equal to 0 in $[1 / 3,2 / 3$ ], and equal to -1 in $] 2 / 3,1[$. Then, $F(u] 0,,2 / 3[)=0$, $F(u] 1 / 3,,1[)=0$ but $F(u] 0,,1[)=1$, so that $A \mapsto F(u, A)$ is not an additive set function.

Remark 1.6. In general, there is no hope for uniqueness of the minimizing set $B$ of the problem $(\mathcal{P})$. Indeed take $\Omega=] 0,1[$, the same function $u$ of Remark 1.5 , and any function $g$ such that $g_{+}=-u$ and $g_{-}=+u$. Then, $\min (\mathcal{P})=1 / 3$ and any set $\left.B=\right] 0, t[$ with $1 / 3 \leq$ $t \leq 2 / 3$ is a minimizer. This phenomenon forced us to consider only the convergence of the signs of suitable subsequences of minimizers. The pointwise convergence of the minimizers is ensured under stronger assumptions on $g$, as the following proposition shows.

Proposition 1.7. Let us assume that for almost every $x \in \Omega$ the function $g(x, \cdot)$ has a unique minimizer $u_{+}(x)>0$ when restricted to the half line $[0,+\infty[$ and a unique minimizer $u_{-}(x)<0$ when restricted to the half line $\left.]-\infty, 0\right]$. Then, given any sequence $\left(\varepsilon_{h}\right)$ converging to 0 and any sequence $\left(u_{h}\right)$ of minimizers of $\left(\mathcal{P}_{\varepsilon_{h}}\right)$, there is a subsequence $\left(u_{h_{k}}\right)$ converging in measure to a function $u \in \mathcal{M}(\Omega)$ such that $u(x) \in\left\{u_{-}(x), u_{+}(x)\right\}$ for any $x$ and the set $\{u>0\}$ minimizes $(\mathcal{P})$.

\section{Proof of the Results}

The proof of Theorems 1.1 and 1.3 essentially relies on the following two propositions. We define

$$
m=\inf \{s: \beta(s) \neq 0\}, \quad M=\sup \{s: \beta(s) \neq 0\} .
$$

Proposition 2.1. Let $\left.\left(\varepsilon_{h}\right) \subset\right] 0,+\infty\left[\right.$ be converging to 0 , and let $\left(u_{h}\right) \subset H^{1}(\Omega), u \in$ $\mathcal{M}(\Omega)$ such that

$$
\limsup _{h \rightarrow+\infty} \frac{u_{h}}{\varepsilon_{h}} \geq M
$$

almost everywhere in the set $\{x \in \Omega: u(x)>0\}$ and

$$
\liminf _{h \rightarrow+\infty} \frac{u_{h}}{\varepsilon_{h}} \leq m
$$

almost everywhere in the set $\{x \in \Omega: u(x)<0\}$. Then,

$$
\liminf _{h \rightarrow+\infty} \int_{\Omega}\left[\varepsilon_{h}\left|\nabla u_{h}\right|^{2}+\varepsilon_{h}^{-3} \beta\left(\frac{u_{h}}{\varepsilon_{h}}\right)\right] d x \geq F(u)
$$

with $F(u)$ defined in (1.4). 
Proof. We may assume, possibly passing to subsequences, that the liminf in the statement is a finite limit, say $L$. Let $I$ be the primitive of $\beta^{1 / 2}$ which vanishes for $t \leq m$, and let $v_{h}=2 I\left(u_{h} / \varepsilon_{h}\right)$. By the chain rule and the inequality $2 a b \leq a^{2}+b^{2}$ we infer

$$
\int_{\Omega}\left|\nabla v_{h}\right| d x=\frac{2}{\varepsilon_{h}} \int_{\Omega} \beta^{1 / 2}\left(\frac{u_{h}}{\varepsilon_{h}}\right)\left|\nabla u_{h}\right| d x \leq \int_{\Omega}\left[\varepsilon_{h}\left|\nabla u_{h}\right|^{2}+\varepsilon_{h}^{-3} \beta\left(\frac{u_{h}}{\varepsilon_{h}}\right)\right] d x .
$$

Since $0 \leq v_{h} \leq c$, it follows that the sequence $v_{h}$ is bounded in $B V(\Omega)$, and we can assume, by Rellich's theorem, that it converges almost everywhere to a function $v \in B V(\Omega)$. Moreover passing to the limit as $h \rightarrow+\infty$ in the foregoing inequality, and recalling the lower semicontinuity of the total variation (see for instance [10], Theorem 1.9), we get $|D v|(\Omega) \leq$ $L$. We need only to show that $F(u) \leq|D v|(\Omega)$. Since $0 \leq v \leq c$, by using the FlemingRishel formula (see [10], Theorem 1.23)

$$
|D v|(\Omega)=\int_{0}^{c} P(\{x \in \Omega: v(x)>t\}, \Omega) d t
$$

we can find $t \in] 0, c[$ such that $B=\{x \in \Omega: v>t\}$ is a set of finite perimeter in $\Omega$ and $c P(B, \Omega) \leq|D v|(\Omega)$. By our assumption on $\left(u_{h}\right)$, for almost every $x$ in the set $\{x \in \Omega$ : $u(x)>0\}$ the sequence $v_{h}(x)$ converges to $c$. In particular, $\{x \in \Omega: u(x)>0\} \subset B$ up to a negligible set. A similar argument shows that $B \subset\{x \in \Omega: u(x) \geq 0\}$ up to a negligible set. Hence,

$$
F(u, \Omega) \leq c P(B, \Omega) \leq|D v|(\Omega) \leq L,
$$

and the proposition is proved.

In the proposition below it will be very useful the so-called coarea formula (see for instance [8], 3.2.12)

$$
\int_{B}|\nabla \varphi| d x=\int_{-\infty}^{+\infty} \mathcal{H}^{n-1}(\{x \in B: \varphi(x)=t\}) d t
$$

which holds for every Borel set $B \subset \mathbf{R}^{n}$ and every Lipschitz function $\varphi$.

Proposition 2.2. For any function $u \in \mathcal{M}(\Omega)$ it is possible to find functions $\left(u_{\varepsilon}\right) \subset H^{1}(\Omega)$ converging to $u$ almost everywhere, such that

$$
\lim _{\varepsilon \rightarrow 0^{+}} \int_{\Omega}\left[\varepsilon\left|\nabla u_{\varepsilon}\right|^{2}+\varepsilon^{-3} \beta\left(\frac{u_{\varepsilon}}{\varepsilon}\right)\right] d x=F(u) .
$$

Moreover, the sequence $u_{\varepsilon}$ is bounded in $L^{\infty}(\Omega)$ if $u \in L^{\infty}(\Omega)$.

Proof. The proof is achieved in three steps. We define the functional

$$
F^{+}(u)=\inf \left\{\limsup _{\varepsilon \rightarrow 0^{+}} \int_{\Omega}\left[\varepsilon\left|\nabla u_{\varepsilon}\right|^{2}+\varepsilon^{-3} \beta\left(\frac{u_{\varepsilon}}{\varepsilon}\right)\right] d x: u_{\varepsilon} \rightarrow u \text { a.e. in } \Omega\right\} .
$$


A diagonal argument shows that the infimum in the definition of $F^{+}$is achieved, and $F^{+}$is lower semicontinuous with respect to the almost everywhere convergence. Then, the statement of the proposition is equivalent to the inequality $F^{+}(u) \leq F(u)$ (the "liminf" inequality in (2.2) follows by Proposition 2.1).

Step 1. We assume that $u$ is bounded, $u \neq 0$ almost everywhere, and there is a bounded open set $C \subset \mathbf{R}^{n}$ with a smooth boundary such that

$$
C \cap \Omega=\{x \in \Omega: u(x)>0\}
$$

We denote by $\tau$ the distance function from $\partial C$, by $C_{\eta}$ the set $\{x \in C: \tau(x)<\eta\}$, and by $K$ a positive number such that $[m, M] \subset[-K, K]$. We want to define $u_{\varepsilon}=a_{\varepsilon} \sigma_{\varepsilon}$, where $a_{\varepsilon}$ and $\sigma_{\varepsilon}$ are functions which fulfil suitable conditions. Let $\psi_{\varepsilon} \in C^{\infty}\left(\mathbf{R}^{n}\right)$ such that $0 \leq \psi_{\varepsilon} \leq 1$ and

$$
\psi_{\varepsilon} \equiv 0 \text { in } C_{\sqrt{\varepsilon}} \quad \psi_{\varepsilon} \equiv 1 \text { in } \mathbf{R}^{n} \backslash C_{2 \sqrt{\varepsilon}} \quad\left|\nabla \psi_{\varepsilon}\right| \leq \frac{2}{\sqrt{\varepsilon}}
$$

We define

$$
a_{\varepsilon}(x)=\psi_{\varepsilon}(x) \int_{\Omega} \frac{|u|(y)}{K \rho_{\varepsilon}^{n}} h\left(\frac{y-x}{\rho_{\varepsilon}}\right) d y+\varepsilon,
$$

where $h$ is any fixed convolution kernel. The functions $a_{\varepsilon}$ are in $C^{\infty}\left(\mathbf{R}^{n}\right)$, are greater than $\varepsilon$, converge to $|u| / K$ almost everywhere, and are equal to $\varepsilon$ on $C_{\sqrt{\varepsilon}}$. In addition, the upper bound on $\left|\nabla \psi_{\varepsilon}\right|$ yields

$$
\lim _{\varepsilon \rightarrow 0^{+}} \varepsilon \int_{\Omega}\left|\nabla a_{\varepsilon}\right|^{2} d x=0
$$

provided $\rho_{\varepsilon}$ converges to 0 slowly enough. Now we turn to the construction of $\sigma_{\varepsilon}$. Let $\eta_{\varepsilon}>0$, $\gamma_{\varepsilon}$ be solutions of the problem

$$
\gamma^{\prime}=\frac{\beta^{1 / 2}(\gamma(t))+\varepsilon^{3 / 2}}{\varepsilon^{3}}, \quad \gamma(0)=-K, \quad \gamma\left(\eta_{\varepsilon}\right)=K
$$

By a change of variables we infer

$$
\eta_{\varepsilon}=\int_{-K}^{K} \frac{\varepsilon^{3}}{\beta^{1 / 2}(t)+\varepsilon^{3 / 2}} d t \leq 2 K \varepsilon^{3 / 2}
$$

The functions $\sigma_{\varepsilon}$ are defined as follows:

$$
\sigma_{\varepsilon}(x)= \begin{cases}K & \text { if } x \in C \text { and } \tau(x) \geq \eta_{\varepsilon} \\ \gamma_{\varepsilon}(\tau(x)) & \text { if } x \in C \text { and } \tau(x)<\eta_{\varepsilon} \\ -K & \text { if } x \notin C .\end{cases}
$$


By (2.5), for $\varepsilon$ small enough the functions $a_{\varepsilon}$ are equal to $\varepsilon$ in $C_{\eta_{\varepsilon}}$ and, setting $u_{\varepsilon}=a_{\varepsilon} \sigma_{\varepsilon}$, we have $\beta\left(u_{\varepsilon} / \varepsilon\right)=0$ outside $C_{\eta_{\varepsilon}}$. By (2.4) we infer

$$
\limsup _{\varepsilon \rightarrow 0^{+}} \int_{\Omega}\left[\varepsilon\left|\nabla u_{\varepsilon}\right|^{2}+\varepsilon^{-3} \beta\left(\frac{u_{\varepsilon}}{\varepsilon}\right)\right] d x=\lim _{\varepsilon \rightarrow 0^{+}} \int_{B_{\varepsilon}}\left[\varepsilon^{3}\left|\nabla \sigma_{\varepsilon}\right|^{2}+\varepsilon^{-3} \beta\left(\sigma_{\varepsilon}\right)\right] d x
$$

where we have set for simplicity $B_{\varepsilon}=C_{\eta_{\varepsilon}}$. Since $|\nabla \tau|=1$ almost everywhere (see for instance [8], 3.2.34), by using the coarea formula (2.1) and our special choice of $\gamma_{\varepsilon}$ we get

$$
\begin{aligned}
& \left.\limsup \int_{\varepsilon \rightarrow 0^{+}}\left[\varepsilon^{3}\left|\nabla \sigma_{\varepsilon}\right|^{2}+\varepsilon^{-3} \beta\left(\sigma_{\varepsilon}\right)\right)\right] d x= \\
= & \limsup \int_{\varepsilon \rightarrow 0^{+}}^{\eta_{\varepsilon}}\left[\varepsilon^{3}\left|\gamma_{\varepsilon}^{\prime}\right|^{2}+\varepsilon^{-3} \beta\left(\gamma_{\varepsilon}\right)\right] \mathcal{H}^{n-1}(\{x \in C \cap \Omega: \tau(x)=t\}) d t= \\
= & \limsup \int_{\varepsilon \rightarrow 0^{+}}^{\eta_{\varepsilon}}\left[2 \gamma_{\varepsilon}^{\prime} \beta^{1 / 2}\left(\gamma_{\varepsilon}\right)+1\right] \mathcal{H}^{n-1}(\{x \in C \cap \Omega: \tau(x)=t\}) d t .
\end{aligned}
$$

Since $\partial C$ is smooth, for $t$ small enough we have $\{x \in C: \tau(x)=t\}=\{y+t \nu(y): y \in$ $\partial C\}$, where $\nu$ is the inner normal to $C$. In particular,

$$
\begin{array}{r}
\mathcal{H}^{n-1}(\{x \in C \cap \Omega: \tau(x)=t\}) \leq \mathcal{H}^{n-1}(\{y+t \nu(y): y \in \partial C, \operatorname{dist}(y, \Omega)<t\}) \leq \\
\leq(1+L t)^{n-1} \mathcal{H}^{n-1}(\{x \in \partial C: \operatorname{dist}(x, \Omega)<t\}) .
\end{array}
$$

In the above formula, $1+L t$ is greater than the Lipschitz constant of the map $y \mapsto y+$ $t \nu(y)$ defined in $\partial C$, and $L$ is a suitable constant depending on the curvatures of $C$. Finally, assembling the previous inequalities we get

$$
\limsup _{\varepsilon \rightarrow 0^{+}} \int_{\Omega}\left[\varepsilon\left|\nabla u_{\varepsilon}\right|^{2}+\varepsilon^{-3} \beta\left(\frac{u_{\varepsilon}}{\varepsilon}\right)\right] d x \leq 2\left(\int_{-\infty}^{+\infty} \beta^{1 / 2}(s) d s\right) \mathcal{H}^{n-1}(C \cap \bar{\Omega})
$$

Step 2. We now remove the regularity assumption on the set $C=\{x \in \Omega: u(x)>0\}$, assuming only that $P(C, \Omega)<+\infty$. By using Proposition 2.16 and Remark 2.13 of [10], it is possible to extend the characteristic function of $C$ to a function $v \in B V\left(\mathbf{R}^{n}\right)$ with compact support such that $0 \leq v \leq 1$ and $|D v|(\partial \Omega)=0$. Let $\left(\rho_{h}\right)$ be a sequence of mollifiers, and let $v_{h}=v * \rho_{h}$; by Sard's theorem, almost every level set of $v_{h}$ is smooth. Moreover, given any $\eta \in] 0,1 / 2\left[\right.$, by the coarea formula $(2.1)$ for every $h \in \mathbf{N}$ we can find $\left.t_{h} \in\right] \eta, 1-\eta[$ such that the set

$$
C_{h}=\left\{x \in \mathbf{R}^{n}: v_{h}(x)>t_{h}\right\}
$$

is smooth and

$$
\mathcal{H}^{n-1}\left(C_{h} \cap \bar{\Omega}\right) \leq \frac{1}{1-2 \eta} \int_{\bar{\Omega}}\left|\nabla v_{h}\right| d x=\frac{1}{1-2 \eta}\left|D v_{h}\right|(\Omega) .
$$


Now we set

$$
u_{h}(x)= \begin{cases}|u|(x) & \text { if } x \in C_{h} \\ -|u|(x) & \text { if } x \in \Omega \backslash C_{h}\end{cases}
$$

Since $v_{h}$ converges to $\chi_{C}$ almost everywhere in $\Omega$, it can be easily seen that $u_{h}$ converges to $u$ almost everywhere. Since $u_{h}$ fulfil condition (2.3), by (2.6) and (2.7) we infer

$$
F^{+}\left(u_{h}\right) \leq \frac{c}{1-2 \eta}\left|D v_{h}\right|(\Omega)
$$

Since $|D v|(\partial \Omega)=0$, the sequence $\left|D v_{h}\right|(\Omega)$ converges to $|D v|(\Omega)=P(C, \Omega)$ (see for instance [10], Proposition 1.15). By letting $h \rightarrow+\infty$ we get

$$
F^{+}(u) \leq \frac{1}{1-2 \eta} F(u)
$$

and the inequality follows by letting $\eta \rightarrow 0$.

Step 3. Let $B$ be a minimizing set in the definition of $F(u)$. Let $u_{h}$ be the functions defined by

$$
u_{h}(x)= \begin{cases}(h \wedge u(x)) \vee-h & \text { if } u(x) \neq 0 \\ 1 / h & \text { if } u(x)=0 \text { and } x \in B \\ -1 / h & \text { if } u(x)=0 \text { and } x \notin B\end{cases}
$$

Since the functions $u_{h}$ are bounded and are nowhere equal to zero, the first two steps yield

$$
F^{+}\left(u_{h}\right) \leq F\left(u_{h}\right)=F(u)
$$

By letting $h \rightarrow+\infty$ and using the lower semicontinuity of $F^{+}$we obtain the desired inequality.

Proof of Theorem 1.1. Let $B$ be a minimizer of $(\mathcal{P})$, and let $a_{h}, b_{h}$ be the functions in Remark 1.2. We define

$$
u_{h}(x)= \begin{cases}b_{h}(x) & \text { if } x \in B \\ a_{h}(x) & \text { if } x \notin B\end{cases}
$$

By Proposition 2.2, for every integer $h$ we can find a sequence $\left(u_{\varepsilon}\right)$ converging to $u_{h}$ almost everywhere, bounded in $L^{\infty}(\Omega)$, and such that

$$
\begin{aligned}
\lim _{\varepsilon \rightarrow 0^{+}} & {\left[\int_{\Omega}\left[\varepsilon\left|\nabla u_{\varepsilon}\right|^{2}+\varepsilon^{-3} \beta\left(\frac{u_{\varepsilon}}{\varepsilon}\right)\right] d x+\int_{\Omega} g\left(x, u_{\varepsilon}\right) d x\right]=} \\
& =F\left(u_{h}\right)+\int_{\Omega} g\left(x, u_{h}\right) \leq c P(B, \Omega)+\int_{\Omega} g\left(x, u_{h}\right) d x .
\end{aligned}
$$


In particular,

$$
\begin{aligned}
\limsup _{\varepsilon \rightarrow 0^{+}}\left[\min \left(\mathcal{P}_{\varepsilon}\right)\right] & \leq \lim _{h \rightarrow+\infty}\left[c P(B, \Omega)+\int_{\Omega} g\left(x, u_{h}\right) d x\right]= \\
& =c P(B, \Omega)+\int_{B} g_{+} d x+\int_{\Omega \backslash B} g_{-} d x=\min (\mathcal{P}) .
\end{aligned}
$$

In order to show the inequality

$$
\liminf _{\varepsilon \rightarrow 0^{+}}\left[\min \left(\mathcal{P}_{\varepsilon}\right)\right] \geq \min (\mathcal{P})
$$

we choose a sequence $\left(\varepsilon_{h}\right)$ converging to 0 such that

$$
\liminf _{\varepsilon \rightarrow 0^{+}}\left[\min \left(\mathcal{P}_{\varepsilon}\right)\right]=\lim _{h \rightarrow+\infty}\left[\min \left(\mathcal{P}_{\varepsilon_{h}}\right)\right]=L
$$

and we assume $L<+\infty$ (the inequality being trivial if $L=+\infty$ ). Let $u_{h} \in H^{1}(\Omega)$ be a minimizer of $\left(\mathcal{P}_{\varepsilon_{h}}\right)$, and let $v_{h}=2 I\left(u_{h} / \varepsilon_{h}\right)$ be as in the proof of Proposition 2.1. The same argument of Proposition 2.1 shows that the sequence $\left(v_{h}\right)$ is bounded in $B V(\Omega)$. Hence, it it not restrictive to assume that $v_{h}$ converges almost everyhwere to a function $v \in B V(\Omega)$. We define

$$
\begin{aligned}
& B_{1}=\left\{x \in \Omega \quad: \limsup _{h \rightarrow+\infty} \frac{u_{h}(x)}{\varepsilon_{h}} \geq M\right\} \\
& B_{2}=\left\{x \in \Omega \quad: \liminf _{h \rightarrow+\infty} \frac{u_{h}(x)}{\varepsilon_{h}} \leq m\right\} \\
& u(x)= \begin{cases}1 & \text { if } x \in B_{1} \\
-1 & \text { if } x \in B_{2} \\
0 & \text { otherwise. }\end{cases}
\end{aligned}
$$

By Proposition 2.1 we get

$$
\liminf _{h \rightarrow+\infty} \int_{\Omega}\left[\varepsilon_{h}\left|\nabla u_{h}\right|^{2}+\varepsilon_{h}^{-3} \beta\left(\frac{u_{h}}{\varepsilon_{h}}\right)\right] d x \geq F(u) .
$$

If $x \in B_{1}$ and $v_{h}(x)$ converges, then necessarily the limit of $v_{h}$ is equal to $c$, and

$$
\liminf _{h \rightarrow+\infty} \frac{u_{h}(x)}{\varepsilon_{h}} \geq M
$$

because $2 I(t)<c$ for all $t<M$. In particular,

$$
\liminf _{h \rightarrow+\infty} u_{h}(x) \geq 0
$$


for almost every $x \in B_{1}$, hence

$$
\liminf _{h \rightarrow+\infty} \int_{B_{1}} g\left(x, u_{h}\right) d x \geq \int_{B_{1}} \liminf _{h \rightarrow+\infty} g\left(x, u_{h}\right) d x \geq \int_{B_{1}} g_{+} d x
$$

A similar argument gives

$$
\liminf _{h \rightarrow+\infty} \int_{B_{2}} g\left(x, u_{h}\right) d x \geq \int_{B_{2}} g_{-} d x .
$$

Moreover, for every $x \in \Omega \backslash\left(B_{1} \cup B_{2}\right)$ the sequence $\left(u_{h}\right)$ converges to 0 , so that

$$
\liminf _{h \rightarrow+\infty} \int_{\Omega \backslash\left(B_{1} \cup B_{2}\right)} g\left(x, u_{h}\right) d x \geq \int_{\Omega \backslash\left(B_{1} \cup B_{2}\right)} g(x, 0) d x .
$$

The last three inequalities yield

$$
\liminf _{h \rightarrow+\infty} \int_{\Omega} g\left(x, u_{h}\right) d x \geq \int_{B} g_{+} d x+\int_{\Omega \backslash B} g_{-} d x
$$

for any Borel set $B$ containing $B_{1}$ and contained in $\Omega \backslash B_{2}$. By taking as $B$ the minimizing set in the definition of $F(u)$, the inequality (2.8) follows by (2.9) and (2.10).

This proves (1.2). The last statements of the theorem can be shown by repeating the same argument leading to (2.9) with an arbitrary sequence $\left(\varepsilon_{h}\right)$. Finally, if (1.3) holds and if the sequence $\left(u_{h_{k}}\right)$ were converging to 0 in a set of positive measure, then the strict inequality in (2.10) would imply a strict inequality in (2.8), that is a contradiction.

Proof of Theorem 1.3. By Proposition 2.1 we infer

$$
\liminf _{\varepsilon \rightarrow 0^{+}} F_{\varepsilon}\left(u_{\varepsilon}\right) \geq F(u)
$$

whenever $u_{\varepsilon} \rightarrow u$ in measure. In fact, sequences converging in measure admit subsequences converging almost everywhere. By Proposition 2.2 we get a sequence $\left(u_{\varepsilon}\right)$ converging to $u$ in measure such that

$$
\lim _{\varepsilon \rightarrow 0^{+}} F_{\varepsilon}\left(u_{\varepsilon}\right)=F(u) .
$$

This proves (see for instance [2]) the $\Gamma$-convergence of $F_{\varepsilon}$ to $F$.

Proof of Proposition 1.7. By Theorem 1.1, we may assume, with no loss of generality, that $\operatorname{sign}\left(u_{h}\right)$ converges almost everywhere to $\chi_{B}-\chi_{\Omega \backslash B}$ for a suitable minimizer $B$ of $\mathcal{P}$. We shall prove that $u_{h}$ converges in measure to the function defined by

$$
u(x)= \begin{cases}u_{+}(x) & \text { if } x \in B \\ u_{-}(x) & \text { if } x \in \Omega \backslash B\end{cases}
$$


Let $\delta>0$ be given; by the assumptions on $g$ there exists $\gamma(x)>0$ such that

$$
\begin{array}{ll}
g(x, t) \geq \gamma(x)+g_{+}(x) & \text { if } t \geq 0 \text { and }\left|t-u_{+}(x)\right| \geq \delta \\
g(x, t) \geq \gamma(x)+g_{-}(x) & \text { if } t \leq 0 \text { and }\left|t-u_{-}(x)\right| \geq \delta .
\end{array}
$$

Therefore, setting

$$
\Omega_{h}=\left\{x \in \Omega: \operatorname{sign} u_{h}(x)=\operatorname{sign} u(x),\left|u_{h}(x)-u(x)\right| \geq \delta\right\}
$$

it is easy to obtain

$$
\int_{\Omega} g\left(x, u_{h}\right) d x \geq \int_{\Omega_{h}} \gamma d x+\int_{\left\{u_{h} \geq 0\right\} \cap B} g_{+} d x+\int_{\left\{u_{h}<0\right\} \backslash B} g_{-} d x .
$$

By Theorem 1.1 and Proposition 2.1 we get

$$
\limsup _{h \rightarrow+\infty} \int_{\Omega} g\left(x, u_{h}\right) d x \leq \int_{B} g_{+} d x+\int_{\Omega \backslash B} g_{-} d x
$$

so that, by (2.11) and (2.12),

$$
\lim _{h \rightarrow+\infty} \int_{\Omega_{h}} \gamma d x=0
$$

Since $\gamma>0$ and $\operatorname{sign}\left(u_{h}\right) \rightarrow \operatorname{sign}(u)$ a.e. in $\Omega$, this implies

$$
\lim _{h \rightarrow+\infty} \operatorname{meas}\left(\left\{x \in \Omega:\left|u_{h}(x)-u(x)\right| \geq \delta\right\}\right)=0
$$

and, since $\delta>0$ is arbitrary, we obtain the convergence in measure of $u_{h}$ to $u$.

Acknowledgements: The third author gratefully acknowledges the Institute for Mathematics and its Applications of the University of Minnesota for its support during the completion of this paper.

\section{References}

[1] L. AMBROSIO: Metric space valued functions of bounded variation. Ann. Scuola Norm. Sup. Pisa Cl. Sci., (to appear).

[2] H. ATTOUCH: Variational Convergence for Functions and Operators. Pitman, Boston, 1984.

[3] S. BALDO: Minimal interface criterion for phase transitions in mixtures of CahnHilliard fluids. Ann. Inst. H. Poincaré Anal. Non Linéaire, 7 (2) (1990), 67-90. 
[4] G. BOUCHITTE: Singular perturbation of variational problems arising from a two-phase transition model. Appl. Math. Optim., 21 (1990), 289-314.

[5] H. BREZIS: Analyse Fonctionelle. Masson, Paris, 1983.

[6] G. BUTTAZZO: Semicontinuity, Relaxation and Integral Representation in the Calculus of Variations. Pitman Res. Notes Math. Ser., 207, Longman, Harlow, 1989.

[7] C. CASTAING \& M. VALADIER: Convex Analysis and measurable Multifunctions. Lecture Notes in Math., 590, Springer-Verlag, Berlin, 1977.

[8] H. FEDERER: Geometric Measure Theory. Springer-Verlag, Berlin, 1969.

[9] I. FONSECA \& L. TARTAR: The gradient theory of phase transitions for systems with two potential wells. Proc. Roy. Soc. Edinburgh, A-111 (1989), 89-102.

[10] E. GIUSTI: Minimal Surfaces and Functions of Bounded Variation. Birkäuser, Boston, 1984.

[11] M. E. GURTIN: On a theory of phase transitions with interfacial energy. Arch. Rational Mech. Anal., 87 (1984), 187-212.

[12] R. V. KOHN \& P. STERNBERG: Local minimizers and singular perturbations. Proc. Roy. Soc. Edinburgh, A-111 (1989), 69-84.

[13] S. LUCKHAUS \& L. MODICA: The Gibbs-Thompson relation within the gradient theory of phase transitions. Arch. Rational Mech. Anal., (to appear).

[14] L. MODICA: The gradient theory of phase transitions and the minimal interface criterion. Arch. Rational Mech. Anal., 98 (1987), 123-142.

[15] L. MODICA: Gradient theory of phase transitions with boundary contact energy. Ann. Inst. H. Poincaré Anal. Non Linéaire, 5 (1987), 453-486.

[16] L. MODICA \& S. MORTOLA: Un esempio di $\Gamma$-convergenza. Boll. Un. Mat. Ital., (5) 14-B (1977), 285-299.

[17] L. MODICA \& S. MORTOLA: Il limite nella $\Gamma$-convergenza di una famiglia di funzionali ellittici. Boll. Un. Mat. Ital., (3) 14-A (1977), 526-529.

[18] N. C. OWEN: Nonconvex variational problems with general singular perturbations. Trans. Amer. Math. Soc., (to appear).

[19] N. C. OWEN \& P. STERNBERG: Nonconvex problems with anisotropic perturbations. Nonlinear Anal., (to appear).

[20] P. STERNBERG: The effect of a singular perturbation on nonconvex variational problems. Arch. Rational Mech. Anal., 101 (1988), 209-260. 
H.S. Dumas, J.A. Ellison and A.W. Sáenz, Axial channeling in perfect crystals, the continuum model and the method of averaging

M.A. Kaashoek and S.M. Verduyn Lunel, Characteristic matrices and spectral properties of evolutionary systems

Xinfu Chen, Generation and Propagation of interfaces in reaction diffusion systems

Avner Friedman and Bei Hu, Homogenization approach to light scattering from polymer-dispersed liquid crystal films

Yoshihisa Morita and Shuichi Jimbo, ODEs on inertial manifolds for reaction-diffusion systems in a singularly perturbed domain with several thin channels

Wenxiong Liu, Blow-up behavior for semilinear heat equations:multi-dimensional case

Hi Jun Choe, Hölder continuity for solutions of certain degenerate parabolic systems

Hi Jun Choe, Regularity for certain degenerate elliptic double obstacle problems

Fernando Reitich, On the slow motion of the interface of layered solutions to the scalar Ginzburg-Landau equation

Xinfu Chen and Fernando Reitich, Local existence and uniqueness of solutions of the Stefan problem with surface tension and kinetic undercooling

C.C. Lim, J.M. Pimbley, C. Schmeiser and D.W. Schwendeman, Rotating waves for semiconductor inverter rings

W. Balser, B.L.J. Braaksma, J.-P. Ramis and Y. Sibuya, Multisummability of formal power series solutions of linear ordinary differential equations

Peter J. Olver and Chehrzad Shakiban, Dissipative decomposition of partial differential equations

Clark Robinson, Homoclinic bifurcation to a transitive attractor of Lorenz type, II

Michelle Schatzman, A simple proof of convergence of the $Q R$ algorithm for normal matrices without shifts

Ian M. Anderson, Niky Kamran and Peter J. Olver, Internal, external and generalized symmetries C. Foias and J.C. Saut, Asymptotic integration of Navier-Stokes equations with potential forces. I

Ling Ma, The convergence of semidiscrete methods for a system of reaction-diffusion equations

Adelina Georgescu, Models of asymptotic approximation

A. Makagon and H.Salehi, On bounded and harmonizable solutions on infinite order arma systems

San-Yih Lin and Yan-Shin Chin, An upwind finite-volume scheme with a triangular mesh for conservation laws

J.M. Ball, P.J. Holmes, R.D. James, R.L. Pego \& P.J. Swart, On the dynamics of fine structure

KangPing Chen and Daniel D. Joseph, Lubrication theory and long waves

J.L. Ericksen, Local bifurcation theory for thermoelastic Bravais lattices

Mario Taboada and Yuncheng You, Some stability results for perturbed semilinear parabolic equations

A.J. Lawrance, Local and deletion influence

Bogdan Vernescu, Convergence results for the homogenization of flow in fractured porous media

Xinfu Chen and Avner Friedman, Mathematical modeling of semiconductor lasers

Yongzhi Xu, Scattering of acoustic wave by obstacle in stratified medium

Songmu Zheng, Global existence for a thermodynamically consistent model of phase field type

Heinrich Freistühler and E. Bruce Pitman, A numerical study of a rotationally degenerate hyperbolic system part I: the Riemann problem

Epifanio G. Virga, New variational problems in the statics of liquid crystals

Yoshikazu Giga and Shun'ichi Goto, Geometric evolution of phase-boundaries

Ling Ma, Large time study of finite element methods for 2D Navier-Stokes equations

Mitchell Luskin and Ling Ma, Analysis of the finite element approximation of microstructure in micromagnetics

M. Chipot, Numerical analysis of oscillations in nonconvex problems

J. Carrillo and M. Chipot, The dam problem with leaky boundary conditions

Eduard Harabetian and Robert Pego, Efficient hybrid shock capturing schemes

B.L.J. Braaksma, Multisummability and Stokes multipliers of linear meromorphic differential equations

Tae Il Jeon and Tze-Chien Sun, A central limit theorem for non-linear vector functionals of vector Gaussian processes

Chris Grant, Solutions to evolution equations with near-equilibrium initial values

Mario Taboada and Yuncheng You, Invariant manifolds for retarded semilinear wave equations

Peter Rejto and Mario Taboada, Unique solvability of nonlinear Volterra equations in weighted spaces

Hi Jun Choe, Holder regularity for the gradient of solutions of certain singular parabolic equations

Jack D. Dockery, Existence of standing pulse solutions for an excitable activator-inhibitory system

Jack D. Dockery and Roger Lui, Existence of travelling wave solutions for a bistable evolutionary ecology model

Giovanni Alberti, Luigi Ambrosio and Giuseppe Buttazzo, Singular perturbation problems with a compact support semilinear term

Emad A. Fatemi, Numerical schemes for constrained minimization problems

Y. Kuang and H.L. Smith, Slowly oscillating periodic solutions of autonomous state-dependent delay equations 

regularity of solutions I

Emanuel Parzen, Time series, statistics, and information

Andrew Majda and Kevin Lamb, Simplified equations for low Mach number combustion with strong heat release

Ju. S. Il'yashenko, Global analysis of the phase portrait for the Kuramoto-Sivashinsky equation

James F. Reineck, Continuation to gradient flows

Mohamed Sami Elbialy, Simultaneous binary collisions in the collinear N-body problem

John A. Jacquez and Carl P. Simon, Aids: The epidemiological significance of two different mean rates of partner-change

Carl P. Simon and John A. Jacquez, Reproduction numbers and the stability of equilibria of SI models for heterogeneous populations

Matthew Stafford, Markov partitions for expanding maps of the circle

Ciprian Foias and Edriss S. Titi, Determining nodes, finite difference schemes and inertial manifolds M.W. Smiley, Global attractors and approximate inertial manifolds for abstract dissipative equations

M.W. Smiley, On the existence of smooth breathers for nonlinear wave equations

Hitay Özbay and Janos Turi, Robust stabilization of systems governed by singular integro-differential equations

Mary Silber and Edgar Knobloch, Hopf bifurcation on a square lattice

Christophe Golé, Ghost circles for twist maps

Christophe Golé, Ghost tori for monotone maps

Christophe Golé, Monotone maps of $T^{n} \times R^{n}$ and their periodic orbits

E.G. Kalnins and W. Miller, Jr., Hypergeometric expansions of Heun polynomials

Victor A. Pliss and George R. Sell, Perturbations of attractors of differential equations

Avner Friedman and Peter Knabner, A transport model with micro- and macro-structure

E.G. Kalnins and W. Miller, Jr., A note on group contractions and radar ambiguity functions

George R. Sell, References on dynamical systems

Shui-Nee Chow, Kening Lu and George R. Sell, Smoothness of inertial manifolds

Shui-Nee Chow, Xiao-Biao Lin and Kening Lu, Smooth invariant foliations in infinite dimensional spaces

Kening Lu, A Hartman-Grobman theorem for scalar reaction-diffusion equations

Christophe Golé and Glen R. Hall, Poincaré's proof of Poincaré's last geometric theorem

Mario Taboada, Approximate inertial manifolds for parabolic evolutionary equations via Yosida approximations

Peter Rejto and Mario Taboada, Weighted resolvent estimates for Volterra operators on unbounded intervals

Joel D. Avrin, Some examples of temperature bounds and concentration decay for a model of solid fuel combustion

Susan Friedlander and Misha M. Vishik, Lax pair formulation for the Euler equation

H. Scott Dumas, Ergodization rates for linear flow on the torus

A. Eden, A.J. Milani and B. Nicolaenko, Finite dimensional exponential attractors for semilinear wave equations with damping

A. Eden, C. Foias, B. Nicolaenko \& R. Temam, Inertial sets for dissipative evolution equations
A. Eden, C. Foias, jection

Michel Chipot and Charles Collins, Numerical approximations in variational problems with potential wells

Huanan Yang, Nonlinear wave analysis and convergence of MUSCL schemes

László Gerencsér and Zsuzsanna Vágó, A strong approximation theorem for estimator processes in continuous time

László Gerencsér, Multiple integrals with respect to $L$-mixing processes

David Kinderlehrer and Pablo Pedregal, Weak convergence of integrands and the Young measure representation

Bo Deng, Symbolic dynamics for chaotic systems

702 P. Galdi, D.D. Joseph, L. Preziosi, S. Rionero, Mathematical problems for miscible, incompressible fluids with Korteweg stresses

703 Charles Collins and Mitchell Luskin, Optimal order error estimates for the finite element approximation of the solution of a nonconvex variational problem

Peter Gritzmann and Victor Klee, Computational complexity of inner and outer j-radii of polytopes in finite-dimensional normed spaces

705 A. Ronald Gallant and George Tauchen, A nonparametric approach to nonlinear time series analysis: estimation and simulation 